\title{
Analytical expressions for the shape of axisymmetric membranes with multiple domains
}

\author{
T. Idema ${ }^{1, a}$ and C. Storm ${ }^{2}$ \\ 1 Department of Physics and Astronomy, University of Pennsylvania, 209 S 33rd street, Philadelphia, Pennsylvania 19104, USA \\ 2 Department of Applied Physics and Institute for Complex Molecular Systems, Eindhoven University of Technology, P.O. Box \\ 513, 5600 MB Eindhoven, The Netherlands
}

Received 5 April 2011 and Received in final form 9 June 2011

Published online: 14 July 2011

(c) The Author(s) 2011. This article is published with open access at Springerlink.com

\begin{abstract}
Based on the Canham-Helfrich free energy, we derive analytical expressions for the shapes of axisymmetric membranes consisting of multiple domains. We give explicit equations for both closed vesicles and almost cylindrical tubes. Using these expressions, we also find the shape of a tube attached to a spherical vesicle. The resulting shapes compare well to numerical data, and our expressions can be used to easily determine membrane parameters from experimentally obtained shapes.
\end{abstract}

\section{Introduction}

Membranes compartmentalize the interior of eukaryotic cells, and separate the inside world from the outside. They are also essential for many cellular processes like signaling, trafficking and nutrient uptake, and provide a platform for physical and chemical interactions between proteins [1]. A key component of many such processes is the shape of the membrane, which is either determined intrinsically by its composition, or by the external influence of proteins and molecular motors. A typical example is endocytosis, where several membrane-associated proteins work together to split off a small vesicle with a specific composition from a large membrane [2-5]. Another example is the extraction of long membrane tubes from organelles like the endoplasmic reticulum by motors walking on the cell cytoskeleton [6]. Both intrinsic membrane shapes and the effect of the application of forces on them have been studied extensively. One line of study focuses on artificial membrane systems consisting of a mixture of cholesterol and two other lipids, resulting in a rich phase behavior, including the separation into liquid-ordered $\left(L_{\mathrm{o}}\right)$ and liquid-disordered $\left(L_{\mathrm{d}}\right)$ domains [7-11]. Such domains have been visualized and studied by many groups in recent years [12-18]. Based on the Canham-Helfrich free energy for lipid bilayer membranes [19,20], and the associated shape equations [21-23], several analytical and numerical results on the relation between the shape and composition of membranes have been obtained on the stability and budding of vesicles with domains [24-26], and recently also for their shapes $[14,17]$.

\footnotetext{
a e-mail: tidema@sas.upenn.edu
}

Another line of study focuses on the application of a point force on a membrane vesicle, resulting in narrow membrane tethers or tubes pulled from the vesicles. In such experiments the surface tension of the membrane in the vesicle is usually controlled using pipette aspiration [27-29]. Tubes are often pulled using micropipettes [30] or optical tweezers [31], but also by molecular motors [32-35]. Several attempts have been made to study the effect of a point force on a large membrane vesicle theoretically [36-39], resulting in analytical expressions for small deformations $[37,38]$ as well as for almost-cylindrical long tubes pulled from these vesicles [39]. Recent experimental results also show sorting of multi-component bilayer membranes due to the curvature imposed by tube pulling [40-43], as well as protein sorting due to their spontaneous curvature [43], and domain nucleation at the neck connecting the tube to its mother vesicle [44]. The shape equations for tubular membranes with multiple domains have been solved numerically [44, 45] and approximate expressions based on linearizations of the associated shape equations have also been found [46].

In this paper we study membranes consisting of two different domains in two specific settings: 1) a closed membrane vesicle and 2) an almost cylindrical tube. As outlined above, both have been studied numerically in the past; here we complement those results by deriving analytical expressions for the shape of the membrane. To do so, we first write the shape equation in terms of a single unknown variable: the tangent angle $\psi$ for vesicles, and the radius $r$ for tubes. In both systems, earlier results are based on linearizations of both $\psi$ and $r$ [14,39]. By first eliminating one of those and only linearizing afterwards, 
we eliminate one linearization step, but still obtain shape equations that may be solved in closed form for realistic boundary conditions. These solutions permit analytical analysis of nontrivial shapes, some of which were previously only accessible with numerical methods. For vesicles, this approach leads to a refinement of our earlier results [14]. For tubes, we retrieve the decaying oscillations found before [37-39,46]. Moreover, we use the analytical expression for the shape of a tubular membrane to obtain an approximation of the shape of a tube connected to a spherical vesicle, complementing the known analytical and numerical results.

\section{Shape equation}

The energy associated with the curvature of a membrane is given by the Canham-Helfrich free energy, which includes all contributions of membrane curvature up to second order $[19,20]$. We assume the membrane to be inextensible and hence the total surface area of the membrane to be conserved, which we ensure by adding a Lagrange multiplier (commonly interpreted as the surface tension $\sigma$ ) to the free energy. We can also add a Lagrange multiplier for the total volume enclosed by the membrane (interpreted as the pressure difference across the membrane $p$ ) and an external force $f$. For a single-component membrane with bending modulus $\kappa$ and Gaussian modulus $\bar{\kappa}$, the free energy is then given by

$$
\mathcal{F}=\int\left(\frac{\kappa}{2}\left(2 H-C_{0}\right)^{2}+\bar{\kappa} K+\sigma\right) \mathrm{d} A+p V-f L,
$$

where $H$ is the mean curvature and $K$ the Gaussian curvature, $A$ and $V$ are the membrane's area and enclosed volume, $C_{0}$ its spontaneous curvature, and $L$ is the extension in the direction in which the force is applied. For a single-component closed membrane, the Gauss-Bonnet theorem tells us that the integral over $K$ is a constant. In multi-component membranes, it integrates to a constant plus a boundary term [47].

It is well known that the infinite tube under external tension is a surface which minimizes the Canham-Helfrich free energy $[37,38,48]$. We will show this below from the shape equation associated with the free energy (1). More directly, we can also use the argument of Derényi et al. [37] to relate the radius and applied force for a stable tube to the membrane's material parameters. For a tube of length $L$ and radius $R$ we find from (1)

$$
\mathcal{F}_{\text {tube }}=2 \pi R L\left[\frac{\kappa}{2}\left(\frac{1}{R}-C_{0}\right)^{2}+\sigma\right]+\pi p R^{2} L-f L .
$$

In equilibrium, the bending modulus (which favors a large radius) and the surface tension (which favors a small area, and hence a small radius) have to balance. We find the equilibrium radius $R_{0}$ and force $f_{0}$ by taking derivatives of $\mathcal{F}_{\text {tube }}$ with respect to $R$ and $L$ and equating them to zero, resulting in

$$
\begin{aligned}
0 & =\bar{\sigma}-\frac{1}{2 R_{0}^{2}}+\frac{1}{2} C_{0}^{2}+\bar{p} R_{0}, \\
\frac{f_{0}}{2 \pi \kappa} & =\frac{\bar{p}}{2} R_{0}^{2}+\frac{R_{0}}{2}\left(\frac{1}{R_{0}}-C_{0}\right)^{2}+\bar{\sigma} R_{0},
\end{aligned}
$$

where $\bar{\sigma}=\sigma / \kappa$ and $\bar{p}=p / \kappa$. We note that eq. (3) is the derivative of eq. (4) with respect to $R_{0}$. For the case $f=0$ these equations were first given by Ou-Yang and Helfrich [21]. For the special case that $p=C_{0}=0$, eqs. (3) and (4) simplify to [37]

$$
\begin{aligned}
R_{0} & =\sqrt{\frac{\kappa}{2 \sigma}}, \\
f_{0} & =2 \pi \sqrt{2 \kappa \sigma} .
\end{aligned}
$$

This simple argument gives the correct values of $R_{0}$ and $f_{0}$, but also has a major shortcoming: the free energy as given in eq. (2) is marginally unstable to the unphysical mode of uniform (de)swelling of the entire cylindrical tube. As was shown by Powers et al. [38], a more careful analysis shows that membrane tubes are in fact stable to arbitrary small perturbations, a result we also find as part of our analysis in sect. 4 .

Another minimizer of the Canham-Helfrich free energy at vanishing force is a sphere. Following the same procedure as above, we write for the free energy

$$
\mathcal{F}_{\text {sphere }}=2 \pi \kappa R^{2}\left(\frac{2}{R}-C_{0}\right)^{2}+4 \pi R^{2} \sigma+\frac{4}{3} \pi p R^{3},
$$

from which we find the equilibrium condition

$$
p R^{2}+2 \sigma R-\kappa C_{0}\left(2-C_{0} R\right)=0 .
$$

Equation (8) was also first given by Ou-Yang and Helfrich [21], who also showed that for low pressures the sphere is a stable solution of the shape equation, although for higher pressures it is not. We will only consider the low-pressure case here. For the case of vanishing spontaneous curvature, eq. (8) simplifies to the condition that the pressure difference across the membrane must be given by the Laplace pressure, $p=-2 \sigma / R$.

From the Canham-Helfrich free energy (1), we can obtain a general shape equation, the solutions of which are the extrema of $\mathcal{F}$. The derivation of this general shape equation is rather lengthy but conceptually straightforward, and was first carried out by Ou-Yang and Helfrich [21]. For axisymmetric shapes, such as our tubes and vesicles, the general shape equation reduces to an ordinary differential equation. We parameterize our shape with the contact angle $\psi(s)$, where $s$ is the arc length (see fig. 1 for the coordinates used). The coordinates $r(s)$ (distance to the rotation axis) and $z(s)$ (distance along the rotation axis) are related to $\psi(s)$ by the geometric relations

$$
\begin{aligned}
& \dot{r}(s)=\cos \psi(s), \\
& \dot{z}(s)=-\sin \psi(s),
\end{aligned}
$$




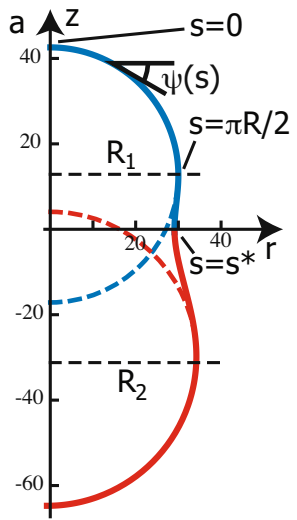

b

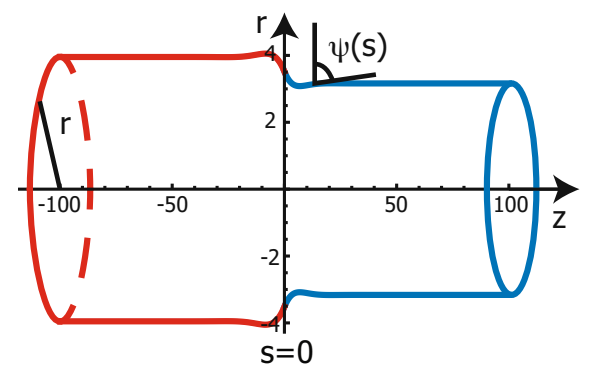

Fig. 1. Coordinate systems used. (a) Coordinates on a closed vesicle. (b) Coordinates on a tubular membrane.

where the dot denotes a derivative with respect to the arc length $s$. We can also express the mean and Gaussian curvatures in terms of $\psi$ (dropping explicit dependencies on $s$ )

$$
\begin{aligned}
2 H & =\dot{\psi}+\frac{\sin \psi}{r}, \\
K & =\frac{\sin \psi}{r} \dot{\psi} .
\end{aligned}
$$

Substituting (11) and (12) into the shape equation derived by Ou-Yang and Helfrich [21] gives us the axisymmetric shape equation

$$
\begin{aligned}
\dddot{\psi}= & -\frac{2 \cos \psi}{r} \ddot{\psi}-\frac{1}{2} \dot{\psi}^{3}+\frac{\sin \psi}{r} \dot{\psi}^{2}+\frac{1}{2} \frac{\sin \psi}{r}\left(\dot{\psi}-C_{0}\right)^{2} \\
& +\frac{1}{2}\left(\frac{\sin \psi}{r}-C_{0}\right)^{2} \dot{\psi}+\frac{1-2 \sin ^{2} \psi}{r^{2}} \dot{\psi}+\frac{\sigma}{\kappa} \dot{\psi} \\
& -\frac{\cos ^{2} \psi+1}{2 r^{3}} \sin \psi+\frac{\sigma}{\kappa} \frac{\sin \psi}{r}+\frac{p}{\kappa} .
\end{aligned}
$$

As was shown by Zheng and Liu [49], eq. (13) can be written as a total derivative, which can be integrated to give an equivalent second-order differential equation for $\psi(s)$

$$
\begin{aligned}
\ddot{\psi} \cos \psi= & -\frac{1}{2} \dot{\psi}^{2} \sin \psi-\frac{\cos ^{2} \psi}{r} \dot{\psi}+\frac{\sin \psi}{2}\left(\frac{\sin \psi}{r}-C_{0}\right)^{2} \\
& +\frac{\cos ^{2} \psi}{r^{2}} \sin \psi+\frac{\sigma}{\kappa} \sin \psi+\frac{p}{2 \kappa} r-\frac{1}{2 \pi \kappa} \frac{f}{r} .
\end{aligned}
$$

Substituting a tubular shape $\left(r=R_{0}\right.$ and $\left.\psi=\pi / 2\right)$ into eq. (14) we again obtain eq. (4), and substituting them into eq. (13) gives eq. (3), in accordance with the observation we made above that eq. (3) is simply the derivative of eq. (4) with respect to the tube radius $R_{0}$. We also find from both eqs. (13) and (14) that in the absence of a force a sphere of any radius $R$ is a solution, provided eq. (8) is satisfied.

In an alternative approach, we can derive the axisymmetric shape equation (14) by writing the energy functional (1) as an integral over a Lagrangian, and performing variational analysis, as detailed by Jülicher et al. [22,23]. The advantage of the latter approach is that it can be easily extended to membranes containing multiple domains, and also gives the boundary conditions at their common edge [23]. Equation (14) holds within the bulk of any continuous piece of membrane, i.e., in any piece where the material constants are the same throughout. At boundaries between coexisting phases 1 and 2, the Lagrangian derivation of (14) gives four boundary conditions: continuity of $r$ and $\psi$, and two more complicated conditions for $\dot{\psi}$ and $\ddot{\psi}$ (expressions below are for a boundary at $s=0$ ):

$$
\begin{aligned}
\lim _{\varepsilon \downarrow 0}\left(\kappa_{2} \dot{\psi}(\varepsilon)-\kappa_{1} \dot{\psi}(-\varepsilon)\right)= & -(\Delta \kappa+\Delta \bar{\kappa}) \frac{\sin \psi_{0}}{r_{0}} \\
& +\kappa_{2} C_{0,2}-\kappa_{1} C_{0,1}, \\
\lim _{\varepsilon \downarrow 0}\left(\kappa_{2} \ddot{\psi}(\varepsilon)-\kappa_{1} \ddot{\psi}(-\varepsilon)\right)= & (2 \Delta \kappa+\Delta \bar{\kappa}) \frac{\cos \psi_{0} \sin \psi_{0}}{r_{0}^{2}} \\
& -\left(\kappa_{2} C_{0,2}-\kappa_{1} C_{0,1}\right) \frac{\cos \psi_{0}}{r_{0}} \\
& +\frac{\sin \psi_{0}}{r_{0}} \tau .
\end{aligned}
$$

Here $\psi_{0}$ and $r_{0}$ are the values of $\psi$ and $r$ at the boundary, $\Delta \kappa=\kappa_{2}-\kappa_{1}, \Delta \bar{\kappa}=\bar{\kappa}_{2}-\bar{\kappa}_{1}$, and $\tau$ is the line tension at the boundary.

\section{Closed vesicles}

We first study closed-vesicle solutions of the axisymmetric shape equation (14). For a single-component membrane, the shape is determined by the ratio of the membrane's surface area and enclosed volume. The optimal solution, i.e., the solution with the minimum total bending energy for a given total membrane area, is a sphere, which also encloses the maximum volume given the total area. For smaller, but fixed, enclosed volumes, solutions include ellipsoids and the biconcave shapes found in red blood cells. Because lipid bilayer vesicles are permeable to water on long time scales, any single-component membrane vesicle left to relax long enough will eventually end up with a spherical shape.

The equilibrium shape of two-component vesicles is not spherical if there is a line tension present at the boundary between the two domains. In that case, the equilibrium shape is determined by a balance between the bending modulus (which drives the system towards a spherical shape) and the line tension (which favors a short boundary line). For approximately equal-sized domains and appropriate values of the material parameters, the resulting shape is a snowman-like vesicle with two almost spherical domains connected by a narrower "waist" or "neck" $[9,12,14]$. We will derive an approximate analytical expression for the shape of these vesicles from the shape equation (14). Since there is no external force in this case, we set $f=0$. Moreover, as in the case of a singlecomponent membrane, for the spherical parts to be stable, eq. (8) must be satisfied in each of the two domains, which means that the pressure, surface tension and spontaneous 
curvature terms in eq. (14) cancel. The resulting differential equation for the contact angle $\psi$ does not depend on any material parameters, which thus only influence the shape because of the boundary conditions. Within the bulk of each of the domains, far away from the boundary, the shape of the vesicle should approximately be a sphere of radius $R$, so $\psi(s) \sim s / R$ and $r(s) \sim R \sin (s / R)$. To meet the boundary conditions, we will perturb these spherical "bulk solutions" and write $\psi(s)=s / R+\delta \psi(s)$. It is not obvious how this perturbation carries over to $r(s)$. Earlier approaches therefore also linearized $r(s)$; instead we eliminate $r(s)$ by isolating it from eq. (14), differentiating the resulting expression and using the geometric relation $\dot{r}=\cos \psi$ (eq. (9)). First rewriting (14), we have

$$
\begin{aligned}
& r^{2}\left(2 \ddot{\psi} \cos \psi+\dot{\psi}^{2} \sin \psi\right) \\
& \quad+r\left(2 \cos ^{2} \psi \dot{\psi}\right)-\left(\cos ^{2} \psi+1\right) \sin \psi=0,
\end{aligned}
$$

from which we get two solutions for $r(s)$

$$
\begin{aligned}
r(s)= & \frac{-\cos \psi \dot{\psi}}{2 \ddot{\psi}+\dot{\psi}^{2} \tan \psi} \\
& \pm \frac{\sqrt{\dot{\psi}^{2} \sec ^{2} \psi+2 \ddot{\psi} \tan \psi\left(1+\cos ^{2} \psi\right)}}{2 \ddot{\psi}+\dot{\psi}^{2} \tan \psi} .
\end{aligned}
$$

Because the sphere is only a solution of eq. (18) when we use the plus sign in front of the square root, we drop the equation with the minus sign. Differentiating the remaining equation with respect to $s$ and using eq. (9), we get a third-order nonlinear differential equation for $\psi$

$$
\begin{aligned}
0= & \left(2 \cos \psi \ddot{\psi} \dddot{\psi}-6 \cos \psi \ddot{\psi}^{2}-\sin \psi \dot{\psi}^{2} \ddot{\psi}+\sec \psi \dot{\psi}\right) \\
& \cdot \sqrt{\dot{\psi}^{2} \sec ^{2} \psi+2 \ddot{\psi} \tan \psi\left(1+\cos ^{2} \psi\right)} \\
& -2 \tan \psi\left(1+\cos ^{2} \psi\right) \dddot{\psi} \dddot{\psi}+2\left(3-4 \sin ^{2} \psi\right) \dot{\psi} \ddot{\psi}^{2} \\
& +\left(\sin ^{2} \psi+\tan ^{2} \psi-2 \sec ^{2} \psi\right) \dot{\psi}^{2} \dddot{\psi} \\
& -\left(1+2 \sin ^{2} \psi\right) \tan \psi \dot{\psi}^{3} \ddot{\psi}-\sec ^{2} \psi \dot{\psi}^{5}
\end{aligned}
$$

We now use the expansion $\psi(s)=s / R+\varepsilon \delta \psi(s)$ and expand up to linear order in $\varepsilon$. In doing so, we assume that the derivatives of $\delta \psi$ are of the same order as $\delta \psi$ itself. Carrying out the expansion, we find a much simpler third-order linear differential equation for $\delta \dddot{\psi}$

$$
0=3 \cos (s / R) \delta \ddot{\psi}+R \sin (s / R) \delta \ddot{\psi},
$$

where $\delta \dot{\psi}(s)=\mathrm{d} \delta \psi(s) / \mathrm{d} s$ and $\delta \ddot{\psi}(s)$ and $\delta \dddot{\psi}(s)$ similarly defined. Equation (20) can be integrated directly, resulting in an expression for $\delta \ddot{\psi}$

$$
\delta \ddot{\psi}(s)=A \csc ^{3}\left(\frac{s}{R}\right),
$$

with $A$ an integration constant which has dimension $1 / R^{2}$, and in which we absorb the expansion coefficient $\varepsilon$. Integrating again, we get

$$
\delta \dot{\psi}(s)=\frac{A R}{2} \log \left[\tan \left(\frac{s}{2 R}\right)\right]-\frac{A R}{2} \frac{\cos (s / R)}{\sin ^{2}(s / R)}+b,
$$

where $b$ is another integration constant. Because the integral of $b$ gives a term that scales with $s$, it gives a con- stant contribution to the term $s / R$ in $\psi(s)$; we therefore set $b=0$. A final integration gives us $\delta \psi(s)$

$$
\begin{aligned}
\delta \psi(s)= & \frac{A R^{2}}{2}\left\{\frac{1}{\sin (s / R)}+i \log \left(\tan \left(\frac{s}{R}\right)\right)\right. \\
& \cdot\left[\log \left(1-i \tan \left(\frac{s}{2 R}\right)\right)-\log \left(1+i \tan \left(\frac{s}{2 R}\right)\right)\right] \\
& \left.+i\left[\operatorname{Li}_{2}\left(i \tan \left(\frac{s}{2 R}\right)\right)-\operatorname{Li}_{2}\left(-i \tan \left(\frac{s}{2 R}\right)\right)\right]\right\} \\
& +d,
\end{aligned}
$$

with $d$ a third integration constant and $\operatorname{Li}_{n}(z)$ the polylogarithm (also known as Jonquière's function), defined as

$$
\operatorname{Li}_{n}(z)=\sum_{k=1}^{\infty} \frac{z^{k}}{k^{n}},
$$

for $z \in \mathbb{C}$. The combination of the two logarithms in (23), as well as that of the two polylogarithms, is real for our region of interest $(0<s<\pi R$, see fig. 1a). The function $\delta \psi(s)$ goes to infinity at both ends of this interval and has a minimum at the center $s=\pi R / 2$. We therefore assume the vesicle to be a perfect sphere for $0 \leq s \leq \pi R / 2$, and given by $\psi(s)=s / R+\delta \psi(s)$ for $\pi R / 2 \leq s \leq s^{*}$, where the boundary with the other domain is at $s=s^{*}<\pi R$. This choice fixes the value of the integration constant $d$ because now $\delta \psi(\pi R / 2)$ should vanish, which gives

$$
d=-\frac{A R^{2}}{2}(1-2 G),
$$

where $G$ is Catalan's constant, with numerical value $\sim$ 0.91596559 .

Having found expressions for $\delta \psi(s)$ and $\delta \dot{\psi}(s)$, we can also find an expression for $r(s)$. To do so, we first expand the geometric relation (9) and then integrate it. As the starting point of the integration we take the same reference point $s_{0}=\pi R / 2$ as we did for $\delta \psi(s)$, at which point $r(s)=$ $R$. We then find

$$
\begin{aligned}
r(s)= & R+\int_{s_{0}}^{s} \cos \left(\frac{s^{\prime}}{R}+\delta \psi\left(s^{\prime}\right)\right) \mathrm{d} s^{\prime} \\
= & R \sin \left(\frac{s}{R}\right)+R\left[\delta \psi\left(s^{\prime}\right) \cos \left(\frac{s^{\prime}}{R}\right)\right]_{s^{\prime}=s_{0}}^{s^{\prime}=s} \\
& -R \int_{s_{0}}^{s} \delta \dot{\psi}\left(s^{\prime}\right) \cos \left(\frac{s^{\prime}}{R}\right) \mathrm{d} s^{\prime} \\
= & R \sin \left(\frac{s}{R}\right)+R \cos \left(\frac{s}{R}\right) \delta \psi(s)-\frac{A R^{3}}{2} \cot \left(\frac{s}{R}\right) \\
& -\frac{A R^{3}}{2} \sin \left(\frac{s}{R}\right) \log \left(\tan \left(\frac{s}{2 R}\right)\right),
\end{aligned}
$$

where we first expanded the cosine and then used partial integration. In exactly the same fashion we also find an expression for $z(s)$

$$
\begin{aligned}
z(s)= & z_{0}+R \cos \left(\frac{s}{R}\right)-R \sin \left(\frac{s}{R}\right) \delta \psi(s) \\
& -\frac{A R^{3}}{2} \cos \left(\frac{s}{R}\right) \log \left(\tan \left(\frac{s}{2 R}\right)\right),
\end{aligned}
$$

where $z_{0}=z\left(s_{0}\right)$. 

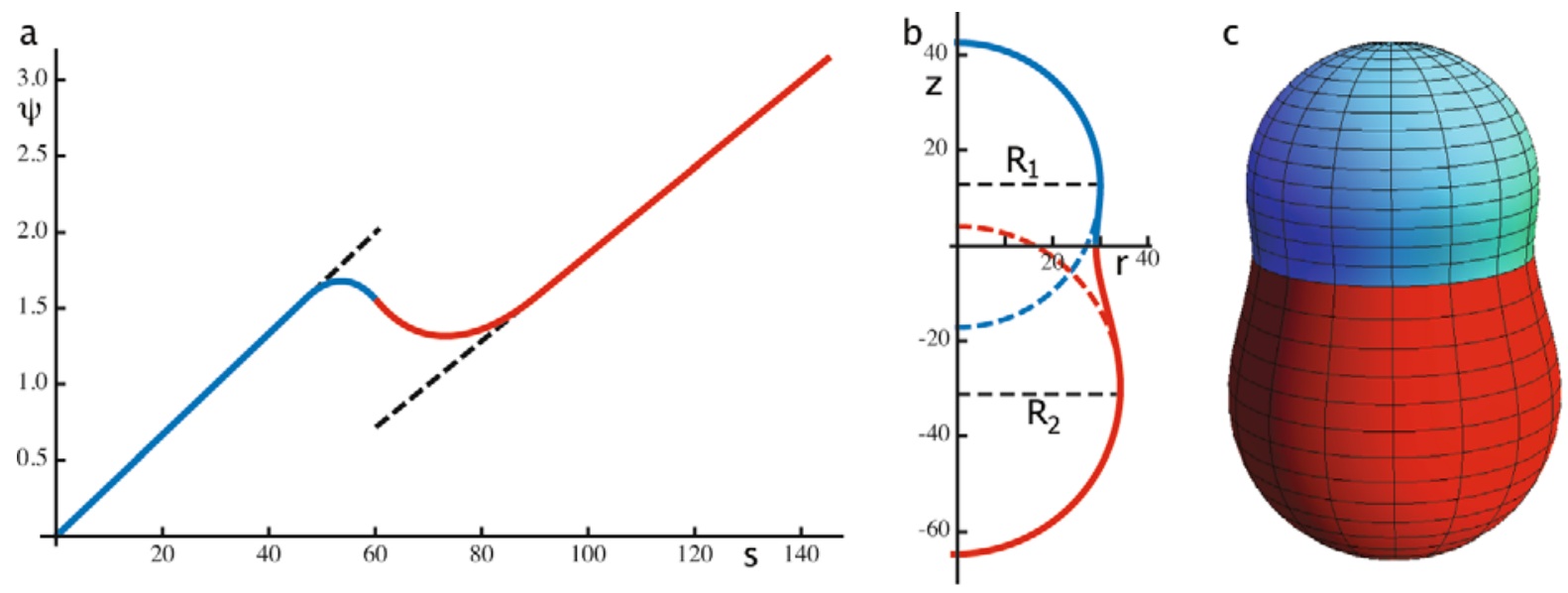

Fig. 2. Shape of a closed vesicle with two domains. Panel (a) shows $\psi(s)$, with dashed lines indicating $\psi(s)$ of a sphere with the same radius. Panels (b) (cross-section) and (c) (revolution plot) show the actual shape of the axisymmetric vesicle, $(r(s), z(s)$ ). Note that the bottom part (with the lower bending modulus) curves towards the top part near the boundary, and that the two parts of the $\psi(s)$ plot are not symmetric.

Using the expressions for $r(s), \delta \psi(s)$ and its derivatives given above, we can find the shape of a vesicle with two domains with different material parameters from the boundary conditions (15) and (16), as well as continuity of $r(s)$ and $\psi(s)$ at the boundary. Writing down the four boundary conditions for the general solution given by eqs. (23) and (26), we find that we have six unknowns: the values of the total arc length $s_{i}^{*}$, the domain radius $R_{i}$ and the dimensionless small parameter $A_{i} R_{i}^{2}$ for each domain $(i=1,2)$. To get a closed system, we can therefore choose any two of the six unknowns, and solve for the other four, although there may not be solutions for every possible choice. Example plots of resulting shapes are given in fig. 2, for which we chose values for the radii $R_{1}$ and $R_{2}$ and solved for the values of $s_{i}^{*}$ and $A_{i} R_{i}^{2}$.

The fact that our shapes are not completely fixed by the set of equations is to be expected, because we did not specify parameters such as the total area or enclosed volume. These are of course fixed in experiment, which allows one to determine (in principle) any two of the six unknown parameters of our system by directly measuring e.g. the domain areas (because of the relation between the pressure, spontaneous curvatures and surface tensions given by eq. (8), the enclosed volume is then fixed). One possible approach is to measure the two values of $s^{*}$ directly by measuring the total arc length of the domains. However, a much simpler and more reliable approach is to measure the values of the two radii $R_{1}$ and $R_{2}$. This can simply be done by fitting a sphere to the upper and lower parts of a snowman-shaped vesicle with two domains. Given these values we are then left with four equations and four unknowns, for any set of the material parameters. There are only two material parameters that play a role in the determination of the shape of the vesicle: the line tension $\tau$ and the difference in Gaussian moduli $\Delta \bar{\kappa}$. We can therefore use this method to fit the shape of the vesicle, and extract value for the line tension and difference in Gaus- sian modulus, as we have done before using a simpler model [14]. Experiments are commonly carried out using Giant Unilamellar Vesicles, or GUVs, which have typical radii of $10-100 \mu \mathrm{m}$. For such large vesicles the assumptions that go into our approach are easily justified. The vesicles are indeed almost indistinguishable from spheres far away from the domain boundary (see e.g. experimental data in $[12,14])$. Moreover, they are smooth near the boundary, and deviate from the spherical shape very little even there, which means that $\delta \psi$ is indeed small. Our approach would break down for much smaller vesicles, for two possible reasons. One is that the deviations may become large compared to the size of the vesicle itself, making a linearized approach invalid. The second reason is more fundamental and has to do with the basic energy balance that results in the snowman shape of bidomain vesicles. In these vesicles, the line tension on the boundary tends to contract the "neck" between the two domain, thus shortening the boundary length. On the other hand the bending energy of both domains tends to expand the neck, making the overall shape vesicle more like that of a sphere and thus lowering the total mean curvature. The tradeoff between these two is characterized by a lenth scale known as the invagination length $\xi=\kappa / \tau$, first introduced by Lipowsky [24]. For domains with a radius much smaller than the invagination length, the bending term in the energy is dominant. The shape of small multi-domain vesicles will therefore not be like our snowman vesicle, but simply a sphere.

\section{Tubes}

Suppose we have a tubular membrane with $L_{\mathrm{o}}$ and $L_{\mathrm{d}}$ domains on it. Within the bulk of those domains, the respective pieces of the tube will approximately be the ideal tube with radii and forces determined by their bending moduli and surface tensions, and given by eqs. (3) and (4) 
for $R_{0}$ and $f_{0}$. For a patterned tube to be stable, the forces in both domains have to be equal. For the special case that $p=C_{0,1}=C_{0,2}=0$ this gives two simple observations; first that the simple relation

$$
\kappa_{\mathrm{o}} \sigma_{\mathrm{o}}=\kappa_{\mathrm{d}} \sigma_{\mathrm{d}}
$$

holds, and second that, if $\kappa_{\mathrm{O}} \neq \kappa_{\mathrm{d}}$, then the radii of the different tube domains are different. Close to the boundary between the two domains the shape will therefore deviate from the cylindrical tube. Shapes for the tube in such boundary regions were first calculated numerically by Allain et al. [45], and more recently also by Heinrich et al. [44]. An approximate analytical expression for the deviation of a tube from a perfect cylinder, for instance because of the presence of a domain boundary, was first given by Božič et al. [39]. For completeness we briefly reproduce their argument here, where for simplicity we again set $p=C_{0}=0$. We first re-parametrize from the arc length $s$ to the $z$-coordinate using eq. (10), and next expand $r(z)$ around $R_{0}$, writing $r(z)=R_{0}+u(z)$. If we moreover assume that the deviations from $\psi=\pi / 2$ are very small, we can also write $\sin \psi=1$ and $\cos \psi=\frac{\mathrm{d} r(s)}{\mathrm{d} s}=$ $-\sin \psi(z) \frac{\mathrm{d} r(z)}{\mathrm{d} z}=-\frac{\mathrm{d} u(z)}{\mathrm{d} z}$. Denoting derivatives to $z$ by primes, we similarly find $\psi^{\prime}(z)=u^{\prime \prime}(z), \psi^{\prime \prime}(z)=u^{\prime \prime \prime}(z)$, and $\psi^{\prime \prime \prime}(z)=u^{\prime \prime \prime \prime}(z)$. Substituting back into the thirdorder shape equation (13), and taking only terms linear in $u(z)$ and its derivatives, we get the simple equation

$$
R_{0}^{4} u^{\prime \prime \prime \prime}(z)+u(z)=0 .
$$

The solution of eq. (29) is a sum of two exponentially decaying oscillations. As shown by Derényi et al. [37], these two oscillations can be interpreted as being associated with the two ends of a finite tube pulled from a vesicle: one end attached to the vesicle, the other closed. Campelo et al. [46] used these solutions in combination with the boundary conditions (15) and (16) to predict the shape of two-component tubes. One possible complication of this approach is that it involves two linearizations, one for $r(z)$ and another one for $\psi(z)$. As we will show below, a different approach allows us to eliminate one of the linearizations, resulting in a different differential equation for the deviation from the tube, but with similar exponentially decaying oscillations as solutions.

The basis of our approach is that we no longer parameterize the tube with the contact angle $\psi$, but simply by its radius as a function of arc length, $r(s)$, and eliminate $\psi$ from the shape equations, similarly to the way we eliminated $r$ in the previous section. Using the geometric relation (9), we can translate $\psi$ and its derivatives into expressions in $r$ and its derivatives. First taking derivatives of (9), we find

$$
\begin{aligned}
\dot{r} & =\cos \psi, \\
\ddot{r} & =-\sin \psi \dot{\psi}, \\
\dddot{r} & =-\sin \psi \ddot{\psi}-\cos \psi \dot{\psi}^{2} .
\end{aligned}
$$

We can invert these to express functions and derivatives of $\psi$ in terms of $r$ and its derivatives

$$
\begin{aligned}
\cos \psi & =\dot{r}, \\
\sin \psi & =\sqrt{1-\dot{r}^{2}}, \\
\dot{\psi} & =-\frac{\ddot{r}}{\sqrt{1-\dot{r}^{2}}}, \\
\ddot{\psi} & =-\frac{1}{\sqrt{1-\dot{r}^{2}}}\left(\dddot{r}+\frac{\dot{r}^{2}}{1-\dot{r}^{2}}\right) .
\end{aligned}
$$

We substitute (33)-(36) back into the shape equation (14). We then find a nonlinear third-order differential equation in $r$

$$
\begin{aligned}
0= & \dot{r} \dddot{r}+\frac{\dot{r}^{2} \ddot{r}^{2}}{1-\dot{r}^{2}}-\frac{1}{2} \ddot{r}^{2}+\frac{\dot{r}^{2} \ddot{r}}{r}+\frac{\dot{r}^{2}}{r^{2}}\left(1-\dot{r}^{2}\right) \\
& +\frac{1-\dot{r}^{2}}{2}\left(\frac{\sqrt{1-\dot{r}^{2}}}{r}-C_{0}\right)^{2}+\bar{\sigma}\left(1-\dot{r}^{2}\right) \\
& +\left(\frac{\bar{p} r}{2}-\frac{f}{2 \pi \kappa r}\right) \sqrt{1-\dot{r}^{2}}
\end{aligned}
$$

where as before $\bar{\sigma}=\sigma / \kappa$ and $\bar{p}=p / \kappa$. The boundary conditions translate to continuity of $r$ and $\dot{r}$, and

$$
\begin{aligned}
& \lim _{\varepsilon \downarrow 0}\left(\kappa_{2} \ddot{r}_{2}(\varepsilon)-\kappa_{1} \ddot{r}_{1}(-\varepsilon)\right)=(\Delta \kappa+\Delta \bar{\kappa}) \frac{1-\dot{r}_{0}^{2}}{r_{0}} \\
& \quad-\left(\kappa_{2} C_{0,2}-\kappa_{1} C_{0,1}\right) \sqrt{1-\dot{r}_{0}^{2}}
\end{aligned}
$$

and

$$
\begin{aligned}
\lim _{\varepsilon \downarrow 0}\left(\kappa_{2} \dddot{r}_{2}(\varepsilon)-\kappa_{1} \dddot{r}_{1}(-\varepsilon)\right) \\
\quad+\frac{\dot{r}_{0}}{1-\dot{r}_{0}^{2}} \lim _{\varepsilon \downarrow 0}\left(\kappa_{2} \ddot{r}_{2}(\varepsilon)^{2}-\kappa_{1} \ddot{r}_{1}(-\varepsilon)^{2}\right) \\
=-(2 \Delta \kappa-\Delta \bar{\kappa}) \frac{\dot{r}_{0}\left(1-\dot{r}_{0}^{2}\right)}{r_{0}^{2}}-\frac{1-\dot{r}_{0}^{2}}{r_{0}} \tau \\
\quad+\left(\kappa_{2} C_{0,2}-\kappa_{1} C_{0,1}\right) \frac{\dot{r}_{0} \sqrt{1-\dot{r}_{0}^{2}}}{r_{0}}
\end{aligned}
$$

for the second and third derivatives. Here $r_{0}$ and $\dot{r}_{0}$ are the values of $r$ and $\dot{r}$ at the domain boundary, and the subscripts 1 and 2 indicate the two domains.

In general the parts of the tube which correspond to the different domains will have unequal stable radii, and thus the tube gets deformed near the domain boundary. Moreover, even with identical radii, but a nonzero line tension $\tau$ or difference in Gaussian modulus $\Delta \bar{\kappa}$, the boundary conditions will distort the shape near the domain boundary as well. To find the perturbed shapes, we make an expansion of $r(s)$ around the stable radius $R_{0}$ (with the appropriate $R_{0}$ for each domain)

$$
r(s)=R_{0}+\varepsilon \delta r(s) .
$$

Substituting (40) into (37), the $\mathcal{O}(1)$ term reproduces eq. (4) and thus vanishes by construction. Moreover, by 
a

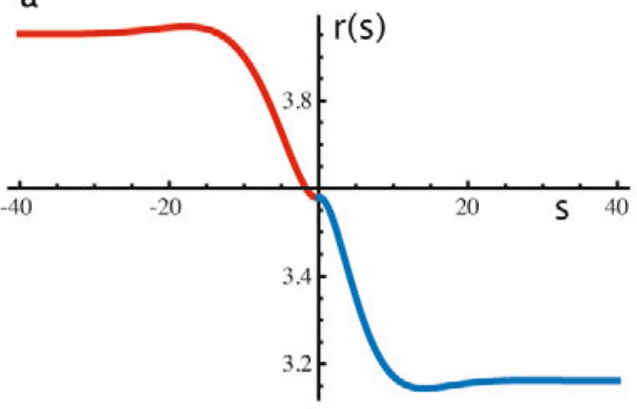

b

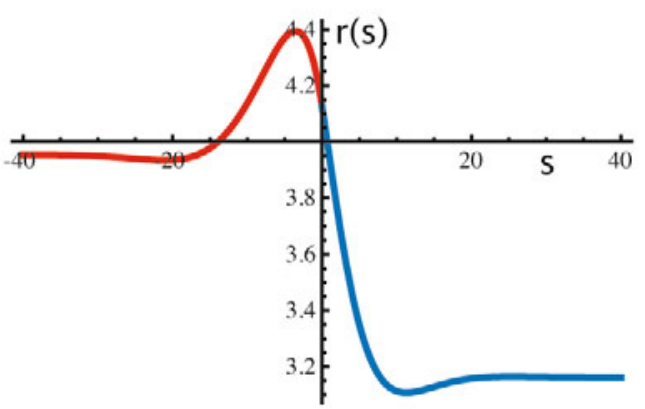

C

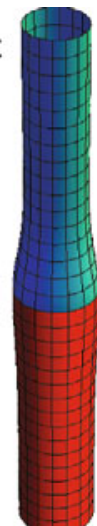

Fig. 3. Example tubes with two domains. (a) Two domains with different material parameters $\left(\kappa_{\mathrm{o}}=1.25 \kappa_{\mathrm{d}}\right)$, but no difference in Gaussian moduli $(\Delta \bar{\kappa}=0)$ and no line tension at the domain boundary $(\tau=0)$. Note the remarkable shape at the boundary with a horizontal tangent line, and the slight deviation of the tube radius just before and after the boundary. These features are also found in numerical solutions of the shape equation (14) [45]. (b) Two domains with different material parameters (again $\kappa_{\mathrm{o}}=1.25 \kappa_{\mathrm{d}}$ ), with a nonzero difference in Gaussian moduli and also a nonzero line tension at the domain boundary. The nonzero boundary contributions allow the high-curvature part associated with the boundary to move into the domain with the lower bending modulus, reducing the total energy. Consequently the tube shows a slight bulge near the boundary. (c) Revolution plot of the actual $(r, z)$ shape of the tube shown in (b). In these plots $p=C_{0,1}=C_{0,2}=0$.

eqs. (3) and (4), the $\mathcal{O}(\varepsilon)$ term vanishes as well, so the $r=R_{0}$ solution is indeed stable, as we claimed in sect. 2 . To second order in $\varepsilon$ we find the equation which will give us the linear perturbation $\delta r(s)$

$$
\left(\frac{1}{R_{0}^{4}}+\frac{\bar{p}}{R_{0}}\right) \delta r^{2}+\left(\frac{2 C_{0}}{R_{0}}+\bar{p} R_{0}\right) \delta \dot{r}^{2}-\delta \ddot{r}^{2}+2 \delta \dot{r} \delta \dddot{r}=0,
$$

where $\delta \dot{r}$ represents the derivative of $\delta r(s)$ with respect to $s$, and so on. To simplify eq. (41), we first introduce two dimensionless parameters

$$
\begin{aligned}
\alpha & \equiv 1+\bar{p} R_{0}^{3}, \\
\beta & \equiv-\bar{p} R_{0}^{3}-2 C_{0} R_{0},
\end{aligned}
$$

so we can express (41) as

$$
\frac{\alpha}{R_{0}^{4}} \delta r^{2}-\frac{\beta}{R_{0}} \delta \dot{r}^{2}-\delta \ddot{r}^{2}+2 \delta \dot{r} \delta \dddot{r}=0 .
$$

Equation (44) can be solved by using the ansatz $\delta r(s)=$ $e^{\lambda s}$, which results in a simple fourth-order equation for $\lambda$

$$
\lambda^{4}-\frac{\beta}{R_{0}} \lambda^{2}+\frac{\alpha}{R_{0}^{4}}=0 .
$$

From eq. (45) we find for $\lambda^{2}$

$$
\lambda^{2}=\frac{1}{R_{0}^{2}}\left(\frac{\beta}{2} \pm \frac{1}{2} \sqrt{\beta^{2}-4 \alpha}\right) \equiv \frac{1}{R_{0}^{2}} \rho_{ \pm},
$$

which means that we have four solutions: $\lambda= \pm \sqrt{\rho_{ \pm}} / R_{0}$. The perturbation $\delta r(s)$ is therefore again a sum of two exponentially decaying oscillations. For infinite tubes, the solutions with positive real part represent exponentially growing perturbations, which we discard. The two solutions with negative real part can be combined into a single solution, which is an exponentially damped oscillation.
In the special case that $p=C_{0}=0$, we get $\rho_{ \pm}= \pm i$, so $\lambda=\frac{1}{R_{0} \sqrt{2}}( \pm 1 \pm i)$, and the resulting expression for $r(s)$ is given by

$$
r(s)=\sqrt{\frac{\kappa}{2 \sigma}}+e^{-\mu s}(a \sin (\mu s)+b \cos (\mu s)),
$$

where $\mu=\sqrt{\sigma / \kappa}=1 /\left(R_{0} \sqrt{2}\right.$ ), and $a$ and $b$ (which should both be $\mathcal{O}(\varepsilon)$ ) are set by the boundary conditions. An example plot of a tubular membrane with two domains is shown in fig. 3 .

In the more general case that only $p=0$ (which is often true in experimental setups, because tubes are connected to large vesicles), we can also find simple expressions for $R_{0}, f_{0}$ and $\lambda$. To do so, we write $C_{0}=\gamma / R_{0}$, and find from eqs. (3) and (4)

$$
\begin{aligned}
R_{0} & =\sqrt{\frac{\left(1-\gamma^{2}\right) \kappa}{2 \sigma}}=\left(\frac{2 \sigma}{\kappa}+C_{0}^{2}\right)^{-1 / 2}, \\
f_{0} & =2 \pi \kappa \frac{1-\gamma}{R_{0}}=2 \pi \kappa\left(\sqrt{\frac{2 \sigma}{\kappa}+C_{0}^{2}}-C_{0}\right) .
\end{aligned}
$$

For $\lambda$ we find from eq. (46) after some algebra

$$
\lambda=\frac{1}{R_{0} \sqrt{2}}( \pm \sqrt{1-\gamma} \pm i \sqrt{1+\gamma})
$$

with $R_{0}$ now given by eq. (48). For $r(s)$ we find in this case

$$
r(s)=\sqrt{\frac{\left(1-\gamma^{2}\right) \kappa}{2 \sigma}}+e^{-\mu_{-} s}\left(a \sin \left(\mu_{+} s\right)+b \cos \left(\mu_{+} s\right)\right),
$$

where $\mu_{ \pm}=\frac{1}{R_{0} \sqrt{2}} \sqrt{1 \pm \gamma}$, and $a$ and $b$ as before set by the boundary conditions. 
As shown by eqs. (48) and (49), the effect of a spontaneous curvature is to lower both the equilibrium radius and the equilibrium force of a membrane tube. Moreover, as shown by eq. (51), the presence of a spontaneous curvature does not change the qualitative shape of a tube near a domain boundary, but does increase both the decay length $\left(\mu_{-}\right)$and the frequency $\left(1 / \mu_{+}\right)$of the associated oscillations.

Unlike the closed vesicles, the tubes have no free parameters to adjust, because the equilibrium radii of the two tubes are determined by the material parameters. We thus have four equations (the four boundary conditions) for four unknowns (the amplitudes $a_{i}$ and $b_{i}$ of the sine and cosine part in eqs. (47) and $(51), i=1,2)$. Because the radius of a tube is already small (tens of nanometers), and the deviations of the tubular shape close to the domain boundary are small compared to their radius, they will be hard to detect directly in experiment. Tubes are therefore not a useful experimental tool to determine parameters that only come in as boundary conditions: the line tension $\tau$ and the difference in Gaussian moduli $\Delta \bar{\kappa}$. However, because forces can be measured with high precision, and because many proteins have spontaneous curvatures comparable to the inverse radius of a tube, measurements on tube extension forces are useful potential probes for the effects of spontaneous curvature. Recent experimental results show that proteins can indeed spontaneously sort in an environment with different curvatures, and are moreover facilitating lipid sorting as well $[42,43]$.

We note that our calculations predict that when the spontaneous curvature becomes comparable to the inverse of the tube radius, the oscillations near the domain boundary become large compared to the radius as well. Our results are therefore no longer strictly valid for values of $\gamma$ close to 1 . However, we do observe that as $\gamma$ gets larger, the tube shrinks while the relative importance of the oscillations grows. The oscillations may therefore play an important role in the eventual instability and breakup of multidomain tubes, which would thus occur sooner for tubes with spontaneous curvature (or containing proteins with an appropriate spontaneous curvature) than for tubes for which the spontaneous curvature vanishes.

As an application of the results derived above, we consider the experimentally more realistic case of a finite tube pulled from a large vesicle. Because the radius of the tube (of the order of tens of nanometers) is much smaller than that of the vesicle (typically several tens of micrometers), the tube will hardly perturb the vesicle shape a distance several times the tube radius away from the neck that connects the two. We simply assume the vesicle has a perfectly spherical shape beyond that point and smoothly connect it to the tube. Our approach breaks down for short tubes, and can therefore not be used to describe tube formation, but can be used to obtain the shape of already formed tubes. Moreover, we consider the case that the force applied at the tip of the tube is distributed over a disk-shaped area (as is the case in actual experiments, e.g., when pulling on an attached bead with optical tweezers), rather than a point force. At the tip we therefore impose the boundary condition that the tangent line to the mem- brane in the $(z, r)$ plane must be vertical. At the vesicle end we simply impose that the tube connects smoothly to the vesicle. Because we now impose boundary conditions at both ends of the tube, we need to use all four solutions of eq. (45), where now one exponentially damped oscillation will start at the tube tip (where the force is applied), and the other at the junction with the vesicle, as shown earlier by Derényi et al. [37]. Again specializing to the case that $p=C_{0}=0$, we then obtain

$$
\begin{aligned}
r(s)= & \sqrt{\frac{\kappa}{2 \sigma}}+e^{-\mu s}\left(a_{1} \sin (\mu s)+b_{1} \cos (\mu s)\right) \\
& +e^{-\mu\left(s_{0}-s\right)}\left(a_{2} \sin \left(\mu\left(s_{0}-s\right)\right)+b_{2} \cos \left(\mu\left(s_{0}-s\right)\right)\right)
\end{aligned}
$$

where $s_{0}$ is the total arc length of the tube. Because in our approach we write $r(s)$ as a function of arc length, we can easily connect to any axisymmetric vesicle shape.

We connect our tube to a large vesicle with given bending modulus $\kappa$ and controlled surface tension $\sigma$. We put the boundary between the vesicle and the tube at $s=0$ as before. For given vesicle and tube radii we then have five unknowns: the parameters $a_{1}, a_{2}, b_{1}$, and $b_{2}$ from eq. (52), and the position of the point $s=0$ with respect to some fixed point on the vesicle (typically the opposite pole), the latter following from the condition that the vesicle must be closed. Two unknowns are fixed by the conditions that at the tube tip $\left(s=s_{0}\right)$ : the radius must vanish, so $r\left(s_{0}\right)=0$, as must the tangent angle, so $\psi\left(s_{0}\right)=0$, which gives $\dot{r}\left(s_{0}\right)=-1$ by eq. (34). The remaining three unknowns allow us to impose equality of $r(s)$ and its first two derivatives at the connection point between the tube and the vesicle. In order to also match higher-order derivatives we should use a higher-order expansion for $r(s)$, and also allow for perturbations of the vesicle itself. Matching up to second order we find solutions that compare well to earlier numerical results, in particular also showing a local narrowing of the tube just before it connects to the vesicle. An example plot is shown in fig. 4.

In this paper we have found approximate solutions for the shapes of axisymmetric membranes. In order to find these solutions, we first wrote the shape equation in terms of the single variable appropriate to the shape we are interested in. We then found the approximate shape by linearizing the resulting single-variable equations. Using this approach, instead of directly linearizing the shape equation, we eliminated one linearization step, which is an improvement of earlier results, and makes our results mathematically rigorous. We found shapes for two-component vesicles and tubes, and also the shape of a single-component vesicle-tube system. We have indicated how these results may be used to extract material parameters from experimental measurements on the different systems. The line tension $\tau$ between two domains and the difference $\Delta \bar{\kappa}$ in their Gaussian moduli can be obtained by directly comparing the experimentally observed shape of closed bicomponent vesicles to the expressions we have given here. In particular $\Delta \bar{\kappa}$ is hard to measure by other methods. Measurements on the force-extension curves of membrane 

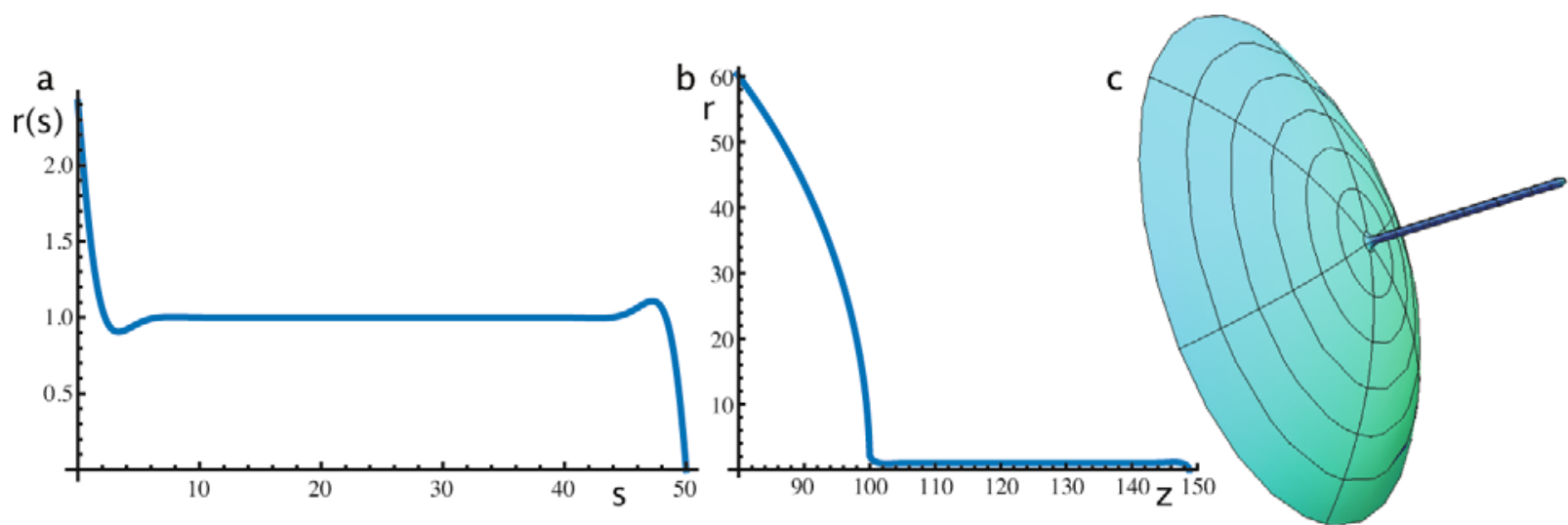

Fig. 4. Example of a tube connected to a spherical vesicle. Here the vesicle radius is $100 R_{0}$ and the tube length is $50 R_{0}$, with $R_{0}$ the radius of the tube; also $p=C_{0,1}=C_{0,2}=0$. (a) $r(s)$ from the neck connecting the tube to the vesicle to the tube tip, showing the characteristic narrowing of the tube at the neck, and widening next to the tip. (b) Large-scale shape of a tube connected to a spherical vesicle in $(z, r)$ coordinates. (c) Revolution plot of panel (b).

tethers can be used as a probe for spontaneous curvature in the membrane, in particular when the membrane contains proteins. We have shown that the presence of a domain boundary in a membrane tube causes decaying oscillations in the tube radius close to the boundary, and how the presence of a spontaneous curvature changes this observation. In particular, we observe that if the spontaneous curvature becomes comparable to the inverse of the radius of the tether, then these oscillations may play a role in the stability of the tether, causing it to break up sooner than one would expect without the presence of the oscillations.

We thank Dr. S. Semrau, Prof. Dr. T. Schmidt and Dr. G.P. Alexander for helpful discussions. This work was supported by funds from the Netherlands Organization for Scientific Research (NWO and NWO-FOM) within the program on Material Properties of Biological Assemblies (FOM-L2601M) and the Rubicon program.

Open Access This article is distributed under the terms of the Creative Commons Attribution Noncommercial License which permits any noncommercial use, distribution, and reproduction in any medium, provided the original author(s) and source are credited.

\section{References}

1. B. Alberts, A. Johnson, J. Lewis, M. Raff, K. Roberts, P. Walter, Molecular Biology of the Cell, 5th edition (Garland Science, New York, NY, USA, 2008).

2. R. Schekman, L. Orci, Science 271, 1526 (1996).

3. J.E. Rothman, F.T. Wieland, Science 272, 227 (1996).

4. J. Bigay, P. Gounon, S. Robineau, B. Antonny, Nature 426, 563 (2003).

5. M. Ehrlich, W. Boll, A. van Oijen, R. Hariharan, K. Chandran, M.L. Nibert, T. Kirchhausen, Cell 118, 591 (2004).

6. C.M. Waterman-Storer, E.D. Salmon, Curr. Biol. 8, 798 (1998).
7. C. Dietrich, L.A. Bagatolli, Z.N. Volovyk, N.L. Thompson, M. Levi, K. Jacobson, E. Gratton, Biophys. J. 80, 1417 (2001).

8. S.L. Veatch, S.L. Keller, Phys. Rev. Lett. 89, 268101 (2002).

9. T. Baumgart, S.T. Hess, W.W. Webb, Nature 425, 821 (2003).

10. S. Munro, Cell 115, 377 (2003).

11. S.L. Veatch, S.L. Keller, Biochim. Biophys. Acta, Biomembr. 1746, 172 (2005).

12. T. Baumgart, S. Das, W.W. Webb, J.T. Jenkins, Biophys. J. 89, 1067 (2005).

13. A.J. Garcia-Saez, S. Chiantia, P. Schwille, J. Biol. Chem. 282, 33537 (2007).

14. S. Semrau, T. Idema, L. Holtzer, T. Schmidt, C. Storm, Phys. Rev. Lett. 100, 088101 (2008).

15. A.R. Honerkamp-Smith, P. Cicuta, M.D. Collins, S.L. Veatch, M. den Nijs, M. Schick, S.L. Keller, Biophys. J. 95, 236 (2008).

16. S. Semrau, T. Idema, T. Schmidt, C. Storm, Biophys. J. 96, 4906 (2009).

17. T.S. Ursell, W.S. Klug, R. Phillips, Proc. Natl. Acad. Sci. U.S.A. 106, 13301 (2009).

18. T. Idema, S. Semrau, C. Storm, T. Schmidt, Phys. Rev. Lett. 104, 198102 (2010).

19. P.B. Canham, J. Theor. Biol. 26, 61 (1970).

20. W. Helfrich, Z. Naturforsch. C 28, 693 (1973).

21. Z.-C. Ou-Yang, W. Helfrich, Phys. Rev. A 39, 5280 (1989).

22. F. Jülicher, U. Seifert, Phys. Rev. E 49, 4728 (1994).

23. F. Jülicher, R. Lipowsky, Phys. Rev. E 53, 2670 (1996).

24. R. Lipowsky, J. Phys. II 2, 1825 (1992).

25. U. Seifert, S. Langer, Europhys. Lett. 23, 71 (1993).

26. U. Seifert, Adv. Phys. 46, 13 (1997).

27. R.M. Hochmuth, E.A. Evans, J.T. McCown, Biophys. J. 39, 71 (1982).

28. R.M. Hochmuth, H.C. Wiles, E.A. Evans, J.T. McCown, Biophys. J. 39, 83 (1982).

29. E. Evans, W. Rawicz, Phys. Rev. Lett. 64, 2094 (1990).

30. E. Evans, H. Bowman, A. Leung, D. Needham, D. Tirrel, Science 273, 933 (1996).

31. G. Koster, A. Cacciuto, I. Derényi, D. Frenkel, M. Dogterom, Phys. Rev. Lett. 94, 068101 (2005). 
32. G. Koster, M. van Duijn, B. Hofs, M. Dogterom, Proc. Natl. Acad. Sci. U.S.A. 100, 15583 (2003).

33. C. Leduc, O. Campàs, K.B. Zeldovich, A. Roux, P. Jolimaitre, L. Bourel-Bonnet, B. Goud, J.-F. Joanny, P. Bassereau, J. Prost, Proc. Natl. Acad. Sci. U.S.A. 101, 17096 (2004).

34. P.M. Shaklee, T. Idema, G. Koster, C. Storm, T. Schmidt, M. Dogterom, Proc. Natl. Acad. Sci. U.S.A. 105, 7993 (2008).

35. C. Leduc, O. Campàs, J.-F. Joanny, J. Prost, P. Bassereau, Biochim. Biophys. Acta, Biomembr. 1798, 1418 (2010).

36. D.J. Bukman, J.H. Yao, M. Wortis, Phys. Rev. E 54, 5463 (1996).

37. I. Derényi, F. Jülicher, J. Prost, Phys. Rev. Lett. 88, 238101 (2002).

38. T.R. Powers, G. Huber, R.E. Goldstein, Phys. Rev. E 65 , 041901 (2002).

39. B. Božič, V. Heinrich, S. Svetina, B. Žekš, Eur. Phys. J. E 6, 91 (2001).
40. A. Roux, D. Cuvelier, P. Nassoy, J. Prost, P. Bassereau, B. Goud, EMBO J. 24, 1537 (2005).

41. H. Jiang, T.R. Powers, Phys. Rev. Lett. 101, 018103 (2008).

42. B. Sorre, A. Callan-Jones, J.-B. Manneville, P. Nassoy, J.F. Joanny, J. Prost, B. Goud, P. Bassereau, Proc. Natl. Acad. Sci. U.S.A. 106, 5622 (2009).

43. A. Tian, T. Baumgart, Biophys. J. 96, 2676 (2009).

44. M. Heinrich, A. Tian, C. Esposito, T. Baumgart, Proc. Natl. Acad. Sci. U.S.A. 107, 7208 (2010).

45. J.-M. Allain, C. Storm, A. Roux, M. Ben Amar, J.-F. Joanny, Phys. Rev. Lett. 93, 158104 (2004).

46. F. Campelo, J.-M. Allain, M. Ben Amar, EPL 77, 38006 (2007).

47. M. do Carmo, Differential Geometry of Curves and Surfaces (Prentice-Hall, Englewood Cliffs, NJ, USA, 1976).

48. E. Evans, A. Yeung, Chem. Phys. Lipids 73, 39 (1994).

49. W.-M. Zheng, J. Liu, Phys. Rev. E 48, 2856 (1993). 